III. Aus dem pathologischen Institut zu Strassburg.

\section{Untersuchungen über die Entstehung der Cysten der Scheide.}

\section{Von Dr. S. Takahasi.}

Obwohl zahlreiche Untersuchungen über die Entstelıung der Scheidencysten von verschiedenen Autoren gemacht worden sind, gehen die Ansicliten hierüber noch sehr auseinauder. In Folge ilurer Aehnlichkeit mit den in anderen Organen vorkommenden Retentionscysten sind einige Autoren [v. Preusclıen, 1) Hennig, ${ }^{2}$ ) Hückel'5)] anzunehmen geneigt, dass sich die Scheidencysten aus den Drüsen der Vaginalschleimhaut bilden. Da aber das Vorkommen von Drüsen in der Scheide anatomisch noch nicht sicher nachgewiesen worden ist, wird diese Ansicht von anderer Seite bestritten [Kaltenbach. ${ }^{4}$ ) Eppinger5)], um so mehr, als die Frage, ob die Schleimhaut der Vagina secernirende Drüisen besitze, noch gänzlich offen ist.

v. Preuschen ${ }^{6}$ ) besclireibt Driisen, welche in doppelter Form mässig tiefe, breite Krypten und schlauchartige Einstïlpungen darstellen. In den anatomischen Lehrbüchern spricht zunächst Henle ${ }^{7}$ ) von uur ausnahmsweise vorkommenden conglobirten Drüsen, während er das Vorhandensein secernirender Drüsen ganz in Abrede stellt. Nach $\mathrm{Hyrtl}^{8}$ ) besitzt dagegen die Scheidenschleimhaut richtige Drüsen, wenn auch nur wenige und einfache Schleimdrüsen. Luschka ${ }^{9}$ ) beschreibt sogar kleinste, ordnungslos zerstreute acinöse Drüschen, besonders am Fornix vaginae sowie zu beiden Seiten des Scheiden einganges.

Andererseits sprechen sich Leydig, ${ }^{10}$ ) Kölliker.11) und Eppinger ${ }^{12}$ ) entschieden für die Drïsenlosigkeit der menschlichen Vagina aus. Auch Foldt ${ }^{13}$ ) sah niemals Drüsen, sondern nur follikelähnliclıe Einlagerungen in lıöchst weclıselnder Anordnung. Auch soll nach der Angabe von Chiarit) schon Haller runde bläuliche Flecke, die wie Vertiefungen aussahen und welchen Mandt ${ }^{15}$ ) die Bezeichnung der Haller'schen Flecke beilegte, auf der dem Uterus zunächst liegenden glatten Scheidenschleimhaut erwähnt haben. Mannigfaltige Untersuchungen, welche von Löwen-

1) v. Prensclien. Ueber Cystenbildung in der Vagina. Virchow's Archiv Bd. LXX, p. 111, 1877.

2) Hennig. Ueber Drüsen in der Vagina. Archiv für Gynåkologie,

Bd. XII, 1877, p. 488.

$\left.{ }^{3}\right)$ Hü ck el. Anatomische Untersuchungen über Colpolıyperplasia cystica. Virch ow's Archiv, Bd. XCIII, p. 204, 1883.

4) Kaltenbacli. Zusammengesetzte Cyste der Scheide. Archiv für Gynäkologie, Bd. V, p. 138, 1873.

5) Eppinger. Emphysema vaginae. Zeitschrift für Heilkunde, Bd. I, p. $369,1880$.

6) v. Preuschen, l. c.

7) Henle. Eingeweidelehre. p. 469, 1875. p. 204 .

8) Hyrtl. Topographische Anatomie. Bd. II, 7. Auflage, 3882,

9) Luschka. Anatomie.

iv) Leydig

11) Kölliker Citat von ${ }^{13}$ ) Eppinger, l. c.

12) Foldt

14) Chiari. Ueber die Gascysten der menschlichen Scheide. Zeitschrift für Heilkunde, Bd. VI, p. 81, 1885, Prag.

i5) Iandt, Citat von Chiari. 
stein ${ }^{1}$ ) angestellt wurden, ergaben ferner, dass die Schleimlant fler Vauina sowohl beim Monschen als bei verschiedenen Hanssïugrethieren Lymplifollikel enthr̈̈lt. Diese solitär stehenden Follikel habeu in der Mehrzahl der Fälle ihren Sitz im obereu glatteu Theil der Vaginalschleimhaut, sie kommen aber auch im unteren faltenreichen Theile vor, und zwar entweder auf der Höhe oder zwischen den Falten. Der Gehalt der Mucosa vaginae an solehen Follikeln erscheint aber individuellen Schwankungen unterworfen, so dass sie sogar mitunter ganz fehlen könuen. v. Preuschen kounte wiederum ans seinen mikroskopischen Präparaten uiemals wirklich geschlossene Follikel auffinden, wohl aber makroskopische Bilder, welche mit Henle'scheı Drüsen übereinstimmen; er gelangte daher mit Eppinger zu der Annahme, dass es sich bei den Henle'schen conglobirten Drïsen um einen pathologischen Befund gehandelt liabe.

Unter deu von mir in pathologischen Institnt untersucliten sechs menschlichen Vaginen kounte ich nur zweimal follikelartige Bildungeu constatiren, niemals aber Drïsen oder drüsenartige Gebilde in irgend einem Prïparate.

In einer Vagina, wo keine Cystenbildung vorhanden, werden solche lymphatischen Follikel in ziemlicher Auzahl im Bereiche des linteren Scheidengewölbes vorgefunden, wälırend in den Schuitteu, die ans dem von der Portio entfernten Theile gefertigt wurdeu, nur einmal ein Follikel beobachtet wird. Auch an der hinteren Lippe der Portio vaginalis zeigen sich ganz ïhnliche Gebilde. Sie liegen unmittelbar nuter dem Epitlıel, welches sich über ilınen keineswegs gegen díe ïbrige Oberfläche erhebt, und sie bestehen aus Zellen, die mit den die Mucosa infiltrirenden Rundzellen gleiche Forı und Grösse haben. Die Follikel sind ferner von dem nmliegenden Gewebe riemlich abgegrenzt und von unregelmässiger Gestalt; sie zeigen in einigen Fäillen mit der Sclıeide communicirende oder abgeschlossene centrale Hölılungen. Maı kann die Follikel bei durchfallendem Lichte sclon mit freiem Auge als Pünktchen dentlich erkenuen. Die Schleimhaut der Vagina ist im Uebrigen stark zellig infiltrirt, ilır Epithel ziemlich gut erlialten und besitzt in der untersten Schicht eine Cylinderzellenreihe, wie dies schon v. Preuschen beschreibt, die Papillen sind lypertrophiscli.

In einer anderen Vagina (IV. Fall s. u.) finden sicli, und zwar iı der nntereu Hälfte der Scheide, ebenfalls derartige Gebilde, in welchen die Zellen ganz dicht gedrängt sind. Sie kommen liier in einer zellig infiltrirten Schleimhaut in Gemeinschaft mit multipleı kleinen Cystclien vor.

Wir lıaben also im ersten Falle dasselbe Verhältuiss vor uns, wie es $J_{,} \ddot{w}$ enstein beschrieben hat. Die Annalıme aber, dass die gefundenen Gebilde normale Lymphfollikel sind, scheint in diesem Falle wegen des gleichzeitig bestehenden entzüudlichen Znstandes der Schleimlant nicht gerechtfertigt zu sein. Chiari²) sagt, dass ilım die lymphfollikelartigen Gebilde, welche er als einen ziemlich häufigeu Befund sowohl bei jüngeren, als bei älteren Individuen constatiren konute, fast immer mit Katarrh der Vagina combinirt zu sein schienen und daher stets den Eindruck pathologischer Neubildungen gemacht hätten.

Wenn anch das Vorkommen mit Epithel verseheuer Drüsen, wie es durch v. Preuschen ${ }^{3}$ ) coustatirt wurde, uicht geläugnet werdeu soll, so muss doch darauf hingewiesen werdeu, dass dieser Befund bis jetzt keine weitere bemerkenswerthe Bestätigung erfahren hat. Eppinger $r^{4}$ ) äussert sich dahin, dass die an Falten so reiche Schleimhant bisweileu deu Drüsen gauz ähuliche Einbuchtungen darstelle, dass sich diese jedoch in keinem Falle als richtige Drüsen erweisen lasseu. Auch ich bin eher geneigt, micl der Eppinger'scheu Anschaunug auzuschliessen, da ich jene von v. Preuschen beschriebeuen Drïsen niemals zu constatireu verwochte. Sehr oft trifft man in der Schleimhant kleine sackartige Einbuchtungen oder tiefe verzweigte Spalten, die mit Epithel überkleidet sind; zuweilen geheu zwischen deu Papilleu Epithelzelllianfeu in die Mucosa hiuein. In den falteureichen Regionen der Scheideuschleimhaut, namentlich am Ausgangstheile, setzen sich nanchmal grosse spaltähuliche Hohlränme tief in die Mucosa hineiu fort, welche bisweilen kleine Nebeuränme besitzen und an der Innenseite, gleich der freieu Schleimhantoberfläche, vou Epithel bekleidet sind. An anderen Orten finden sich sehr complicirte Einbuchtungen, deren Iunenfläche ebenfalls mit Epithel überzogen und deren tiefer gelegene Theile ganz mit Epithelzellen angefüllt sind. Au keiner dieser Buchten siud aber als richtige Ausfülrung's-

1) Löwenstein. Die Lymphfollikel der Schleimhaut der Vagina. (Vorläufige Mitheilung.) Centralblatt für medicinische Wissenschaften, 1871, p. 546 .

\%) Chiari, I. c.

3) r. Preuscheil, I. c.

4) Eppinger, l. c. gänge zu bezeichnende Cauäle vorhanden, auch ist ilıre Anskleidung mit Fpithel keineswegs eine ïberall gleichmässige.

Wie nun die Untersuchungen der Antoren über die Follikel und Drüsen gauz entgegengesetzte Aufstellungeu ergeben, so herrschen auch analoge Meinuugsverschiedenheiten über die Entstehnug der Scheidencysten. v. Preuscheu und Hü ckell) führej die Cystembildung anf die Retention des Drüseninhaltes zurück. während Schroeder ${ }^{2}$ ) die Möglichleit hinstellt, dass die Luftcysteu der Scheide aus deu vou Luschka und Henle zuerst beschriebenen Follikeln so eutständen, dass der klare seröse Inhalt sich später in eiuen gasförmigen uuwandle. Eine andere Entstehnugsart der Luftcysten, uämlich diejenige aus den Lymphgefässen, wird von Spiegelberg, $\left.{ }^{3}\right) \mathrm{Klebs}$ ) und $\mathrm{Chiar^{5 }}$ ) angenommenl. Chiari behanptet, dass sich die Gascysten il präformirten, aber patloologisch dilatirten Räumen des Lymphsystems entwickeln, und dass diese Ränme sich als Lymphcapillaren erweiser lassell, welche mit gewucherten und zum Theil zu Riesenzellen umgewandelten Endothelien erfüllt siud. Ruge $e^{6}$ ) und Eppiuger fanden an den Luftcysten keine selbstständige Wandung und nelımen demnach einen interstitiellen Sitz derselben all. 'Tschudowsky) erklärte eine elastische Geschwulst, welche drei Centimeter vom Introitus vaginae an der linken Seite sass, als eine extraperitoneale apoplectische Cyste.

Wincke19) stellt für die Vaginalcysten mit Rücksiclıt auf ilıre Entstehung drei Arten auf: a) die Schleimliantcysten der Scheide. Cystides mncosae, die aus offenen oder geschlossenen Follikehi hervorgingen; b) die interstitiellen, submucösen oder in der Fibromuskulärschiclıt gelegenen, und c) die subseröseu, oben in dem perivaginalen Biindegewebe nnter dem Peritonenm, unten zwischell Vaginil und Rectun gelegeuen.

In Folgenden will ich nun die Ergebnisse meiner Untersuchungen berichten, welche an fünf im pathologischen Institut voul Herm Professor v. Reckliuglaausen beobachteten Fällen von Schejdencysten unter seiner Aufsiclit und Leitung, wofür ich miclı ihım zum besten Danke verpflichtet fühle, vorgenommeu wurdell. Diese Präparate warell, bevor ich sie bekam, längere Zeit in Mïll er'scher Flüssigkeit und hierauf in Alkohol anfbewahrt worden.

I. Fall. Das Präparat ist schon drei Jahre alt, besteht, aus dell äusseren Genitalien, der Scheide, dem sehr grossen puerperalen Uterus nund der Haruröhre mit einem kleinen Stück der Harıblase. Die Etiquette des das Präparat enthaltenden Gefässes lantet: Metrophlebitis. Lympliallgioitis. Thrombose der Vella spermatica interıa.

An der vorderen Wand der Scheide, und zwar $3,5 \mathrm{~cm}$ voul Introitıs, sitzt eine runde, etwa wallnussgrosse Cyste. Die denl Scheidenrohr zngekelırte freie Wand der Cyste ist kaum 1 lum dick: die Schleimhaut der Vagina ist im Ganzen glatt und bietet sonst nichts Besonderes dar.

Die dem Vaginalrolır zugekelırte Wand der Cyste besteht aus Schleimhant mit einem kleinen Theile der Muscularis; das Epitlel ist hier gut erlialten, die Papillen sehen wie abgeplattet aus. Die Wand ist im Verlı̈̈ltniss zur übrigen Schleimhaut bedeutend dünner. Die übrigen Theile der Cyste liaben keine besondere Wandung, sondern sincl einfach durch Muskelfaserstränge begrenzt.

Die Innenfläche der Cyste ist überall glatt; sowohl all der oberell (freien) Wand, als an denl übrigen Stellen filldet man epitleliale Auskleidung, clas Epithel ist aber nicht überall von gleicher Form: bald findet man geschichtetes Pflasterepithel, welches in der untersten Selicht Cylinderzellen besitzt, bald ein einschichtiges Cylinderepithel. Stellenweise liegt eine homogene Masse auf dem Epithelium, - welches dann immer geschichtet ist, fehlt aber auf dem einfachen Cylinderepithel. Im Grunde der Cyste zeigt die Iunenfläche verschiedene Formen der Einstülpungen, welche ebenfalls mit dem genannten Epithel überkleidet sind, zuweilen haben sie die Gestalt eines Canals, welcher parallel der Cysteninnenfläche verläuft ind mit einfachem Cylinderepithel ausgekleidet ist.

An einer Stelle der Schleimhaut zeigen sich unter dem Mikroskop cystöse Răume, deren grösster sich unterlialb des Epithels bis in die Submucosa erstreckt, indem er siclı in der Tiefe allmählich erweitert. Seine Höhle ist mit anscheinend schleimiger Masse gefüllt; eine epitheliale Auskleidung, sowie eine selbstständige Wand felllt aber sowolıl dieser, wie den anderen kleinerell Cystell.

Das Epithel der bedeckenden Schleimhaut besitzt in der untersten Schicht eine Cylinderzellenreibe; die Gefässe zeigen sich stark erweitert.

II. Fall. Eine schrotkorngrosse, runde Cyste sitzt all der hinteren Wand nahe dem Introitıs. Sie liegt ganz oberflächlich in der Mucosa, die

1) Hückel, l, e.

$\left.{ }^{2}\right)$ Schroeder. Lufteysten ill der Scheidenschleimhaut. Arehiv für klinische Medicin, Bd. XIII, p. 538, 1874.

3) Spiegelberg (Citat von Chénevière. Einige Fälle von Colpo-

4) Klebs

5) Chiari, l, c.

6) Ruge. Zum Bau der Luftcysten der Scheide. Zeitschr. f. Geburtshülfe und Gynäkologie, Bd. II, 1878.

7) Eppinger, l. e.

s) Tschudowsky. Zur Lehre von den Cysten der Scheide. Central blatt für Gynäkologie, No. 16, 1879, p. 408.

$\left.{ }^{9}\right)$ Winckel. Ueber die Cysten der Scheide ete. Archiv für Gynäkologie, Bd. II, 1871, p. 402. 
sehr dünne obere Wand besteht aus der Schleimhaut, die Papillen sind hier von normaler Grösse. Die Innenfläche der Cyste ist mit einer aus platten Zellen gebildeten Zellenschicht überkleidet, welcle ziemlich scharf von dem ımgebenden Bindegewebe abgegrenzt ist. Cylinderzellen konnten in dieser Schicht nicht aufgefunden werden. In der Cyste ist ein fester Körper enthalten, welcher concenirische Schichtungen und radiär verlaufende Streifingenl zeigt, offenbar eine geronnene Colloidmasse.

III. Fall. Auf der linken Seite der Scheide nahe dem Introitus finclet sich eine bohnengrosse, mit einer centralen Depression versehene Cyste. Anf dem Durchschnitt ist die Cyste durch eine Scheidewand in zwei Höhlen getheilt; das Septum besteht aus elastischen Faserzügen der Mucosa, in deren Oberfläche die Cysten ihren Sitz nehmen. Die obere Wand derselben ist dünn 1nd zeigt ein ähnliches Verhältniss wie im vorigen Falle. Die lmuenfläche der Cyste ist nicht gleichmässig mit einem Epithel überzogen, man findet vielmehr nur stellenweise Zellhaufen, welche mit dem geschichteten Pflasterepithel Aehnlichkeit besitzen. Epitheliale Zellen sind dagegenl zuweilen in einer homogenen Masse, welche auf der Innenfläche angetroffen wird, eingeschlossen. Als Inhalt der Cyste findet sich eine klare Flüssigkeit, die beim Einschneiden herausfliesst.

IV. Fall. Die Schleimhant der Scheide ist glatt, an der hinteren Wand der unteren Hälfte sieht man zahlreiche stecknadelkopfgrosse graue Punkte, welche entweder einzeln stehen oder zu mehreren gruppirt sind, und deren Oberfläche eine centrale Depression zeigt. Auf dell Schnitten erwiesen sie sich als Bläschen ohne Inhalt und mit âusserst dünner selbstständiger Wand. Ausser diesen Gebilden kommen noch kleinere, und zwar verschieden grosse cystöse Räume in der Mucosa vor, welche aber keine Prominenzen auf der Schleimhaut verursachen. Ferner findet man in der Oberfäche der Mucosa im Vergleich mit den vorigen noch kleinere Heerde von Zellanlı̈̈ufungen, welche den Lymphfollikeln ähnlich sind. Die Schleimhautpartie über denselben erscheint bisweilen etwas erhaben. Die bindegewebigen Fasern sind um die Cysten herum dicht aneinander gelagert, so dass sie anscheinend eine wandartige Begrenzung darstellen. Die Höhleı der Cysten sind mit płatten Zellen in sehr verschiedener Weise gefüllt; je grösser die Cysten sincl, desto mehr nähert sich die Anordnung der Zellen dem geschichteten Pflasterepithel, wobei dasselbe an der ganzen Peripherie der Höhlung fast die gleiche Dicke hat. Die kleineren, nur mikroskopisch erkennbaren Cystehen sind beinahe ganz von Zellen angefüllt, ja man findet in einzelnen nur einen spaltförmigen Raum inmitten der zelligen Gebilde. Die Zellen sind im Ganzen Ingleich gross, aber stets mit deutlichem Kern versehen. Die Schleimhaut der Scheide ist zellig infiltrirt, diese rundzellige Infiltration ist in der Umgebung der Cysten und der Follike] besonders stark ausgeprägt; die Papillen sind sehr hypertrophirt, an vielen Stellen fehlt das Epithelium.

V. Fall. Scheide und Uterus von einem Neugeborenen. An der linken Seite des hinteren Seheidengewölbes findet sich eine erbsengrosse Cyste, die zum Theil über die seitliche Wand zum vorderen Gewölbe hinüberreicht. Die Cyste zeigte Fluctuation, beim Einschneiden entleerte sich ein klarer flüssiger Inhalt; auf der freien Wand macht sich eine centrale Depression bemerkbar. Die Cyste hat ihren Sitz in der Mucosa, ist durch ziemlich regelmässig gelagerte elastische Faserzüge, welche aber hierbei keine eigene Wandung herstellen, ron dem umliegenden Gewebe abgegrenzt. An der Uebergangsstelle der Scheidenschleimhaut in die die freie Wand der Cyste bildende Schleimhautpartie finden sich Faserzüge der Muscularis mucosa Die Innenffäche der Cyste zeigt überall warzenartige Erhebungen, die entweder einzeln stehen oder zll zweien gruppirt sind und schon makroskopisch beim durchfallenclen licht eine feine, sammetähnliche Anordnung erkennen lassen. Sie bestehen einfach aus elastischen Fasern der Muresa. Sowohl die Spitzen, als auch die Zwischenräume sind mit Epithelium ïberzogen, welches je nach der Lage bald ein geschichtetes Pflasterepitlel, bald ein einfaches Cylinderzellenepithel darstellt. Es gelingt hier aber nicht, in der untersten Schicht jenes Pflasterepithels eine Cylinderzellenreihe zu erkennen. Die Structur der Schleimhaut ist im Vergleich mit den anderen Fällen viel feiner, das Epithel ist stellenweise sehr hoch geschichtet, die Papillen sind bisweilen sehr gestreckt und nicht dentlich erkennbar.

Ueberblicken wir die oben citirten Befunde der füılf Fälle, so liaben wir es mit Cysten zu thull, welche keine eigentliche Wand besitzen, entweder ganz in der Mucosa (II, III, IV, V) oder schon in der Muscularis (I) gelegen und an der Innenfläche mit Schichten von Epithelzellen überzogen sind. Die Cysten kamen mit einer einzigen Ausiahne (IV) nur einzeln vor, und zwar ausser dem Fall V nur in der unteren Hälfte der Scheide; ihre Grösse variirt von der eines Stecknadelkopfs (IV) bis zu der einer Wallnuss (I). Die in grosser Anzahl auftretenden kleinen Cysten im IV. Falle stimmen ihres oberflächlichen Sitzes, ihrer Grösse und der Gruppirung wegen mit der von $\mathrm{W} \mathrm{in} \mathrm{ckel}^{1}$ ) beschriebenen Colpohyperplasia cystica überein. Ein kleiner Unterschied liegt aber darin, dass wir in den Cysten keine eigenartige Wand, auch kein regelmässig kleinzelliges Pflasterepithel oder Endothel mit glatter Fläche nachweisen konnten, wie dieses Winckel in seinen Fällen gefunden liat. Das Vorkommen einer angeborenen Cyste, wovon wir hier ein Beispiel (V. Fall) besitzen, hat Winckel ebenfalls beobachtet; die Cyste befand sich an dem linken Theile der vorderen und seitlichen Scheidenwand und liatte die Harnentleerung erheblicl gelindert: sie enthielt milchig-käsige Flüssigkeit, in welcher sich mikroskopisch zahlreiche Plattenepithelien, sehr reichliches Fett, aber kein Cholestearin nachweisen liessen. Breisky ${ }^{2}$ ) erwähnt eine haselnuss-

1) Winckel. l. c.

2) Breisky. Correspondenzblatt für Schweizer Aerzte. 1875. No. 14, 15. Juli, p. 430 . grosse Cyste bei einem 7 monatlichen Kinde unmittelbar hinter dem Hymen. Ruge ${ }^{1}$ ) hat eine gut erbsengrosse Cyste dicht an der Portio beirn Neugeborenen beobachtet. Beziiglich der Aehnlichkeit seines Falles mit dem unserigen sei noch erwähnt, dass die Cyste im Innern mit einem einschichtigen Cylinderepithel besetzt war.

Das die Innenfläche der Cyste überkleidende Epithel hat in zwei (I und V) unserer Fälle eine grosse Aehnlichkeit mit dem der Scheidenschleimhaut gezeigt. Es ist hierbei bemerkenswertll, dass sich im I. Falle eine Schicht von Cylinderzellen, so wie sie als die unterste Schicht des gewöhnlichen Schleimhautepithels auftritt (vergl. Fall I, ferner v. Preuschen's Befund l. c.), in gleicher Beschaffenheit unter dem geschichteten Epithel der Cysteninnenfläche wieder findet, bisweilen sogar als ein einziger Ueberzug der letzteren vorkommt. Bei dem Fall V kann allerdings keine Cylinderzellenschicht ill dem Schleimhautepithel nachgewiesen werden, aber der epitheliale Ueberzug sowohl auf der Mucosa als auf der Cysteninnenfläclie bietet sonst eine vollkommene Uebereinstimmung dar. In denl übrigen Fällen ist das Verhältniss etwas alıders.

Wenn wir auch über die Herkunft des Cystenepithels keinen bestimmten Ausspruch geben wollen, so erscheint doch in den Fällen I und V die Annahme vollkommen gerechtfertigt, das das die Innenfläche der Cysten überkleidende Epithel aus dem Epithel der Scheidenschleimhaut hervorgegangen ist. Dass ein Theil des Schleimhautepithels in einell Hohlraum gelangt, welcher abgeschlossen wird, lässt sich nur begreifen, wenı ein Verkleben der in der Vagina stets vorhandenen Schleimhautfalten stattfindet. Winckel hebt für eine solche Mögliclıkeit die Beschreibung von Langer hervor, dass die Cysten durch die Verklebung der Deffnung einzelner Buchten und Säcke, welche durch die zahlreichen, ziemlich dicht stehenden, hyperplastischen Schleimhautpartieen gebildet werden, und durch darauf folgende Ansammlung des Secretes entstehen können -- eil Vorgang, der bei acuter Schwellung der hyperplastischen Stellen und starker Secretion, d. h. bei acutem Vaginalkatarrh, wohl möglich ist. Jedoch spricht der tiefe Sitz der Cyste in der. Muscularis im Falle II gegen diese Annahme, da die Falten oder Krypten auf die Mucosa beschränkt sind. Wäre diese Cyste nach der obigen Art entstanden, so müsste dieselbe allseitig von dem Gewebe der Nucosa begrenzt sein; alleiu die Untersuchung zeigte, dass die Cyste zwischen den Muskelfasern sicl gebildet hatte. Winckel giebt ferner an, dass auch solche Cysten vorkommen, deren Wand aus der ganzen Dicke der Schleimhaut und wenigstens einem Theile der fibromuskulären Schicht der Scheide besteht. Diese hätten gar keine besondere Kapsel, sondern wären nur durch eine Dehiscenz der Vaginalwand in Folge eines Ergusses zu Stande gekommen an einer Stelle, wo der diffusell Ausbreitung desselben rings umher Hindernisse erwachsen. Er bringt solche Cysten unter die Rubrik der interstitiellell. ${ }^{2}$ ) Null spricht unser Fall woll für die interstitielle Entstehung, aber dabei ist die Herkunft des die Innenfläche der Cyste iiberkleidenden Epithels schwer zu deuten. Kaltenbach ${ }^{3}$ ) hat ein geschichtetes kleinzelliges Pflasterepithel an der Innenfläche einer auf der hinteren Vaginalwand sitzenden Cyste beobachtet, welche nach seiner Allgabe aus Zellenanhäufungen in der Submucosa entstanden war. Bezüglich der Frage, wie diese Epithelbildung zu Stande gekommen ist, giebt der Autor keine nähere Auskunft.

Hennig ${ }^{4}$ erwähnt den Befund einer der Localität und der Grösse nach mit unserem Falle (I) ganz übereinstimmenden Cyste, welche innen mit einer feil gestichelten (?) Schleimhaut ausgekleidet war; die obere Wand der Cyste war innig mit der Harnröhre verwachsen. Hennig führt die Entstehung der Cyste auf die im vorderen Drittel der Scheide vorkommenden Schleimbälge zalliick.

So lange aber der sichere Nachweis der secermirenden Drüisen in der Vagina anatomisch noch nicht festgestellt ist, wird die Annahme über die Entstehung der Cysten aus diesen nicht ganz begrïndet erscheinen. Noch unwahrscheinlicher aber wäre die Entstehung der Cysten von den Ausfiihrungsgängen der Wolff'schen Körper [G. Veit, ${ }^{5}$ ) Baumgarten $\left.{ }^{6}\right)$ ] wegen der fast gleichen Häufigkeit des Vorkommens sowohl an der vorderen als an der hinteren Wand (nach Winckel 57,5:42,4\%). Schliesslich giebt es noch die eine Möglichkeit, , dass die Verklebung einer Faltenöffnung der Schleimhaut eine Secretansammlung in dem abgeschlossenen Raume herbeiführe und in dieser Weise eine Cystenbildung veranlasse". Fiir die angeborene Cyste (Fall V) scheint eine solche Entstehungs-

1) Ruge. l. c.

2) Siehe seine Eintheilung I. c.

3) Kaltenbach. I. e.

4) Hennig. I. c.

5) G. Veit. Citat von Schroeder (Handb. d. Krankheiten der weibl. Geschlechtsorgane 1887, p. 499). 1887.

6) Baumgarten. Ueber Vaginalcysten. Virchow's Archiv, Bd. 107, 
Weise noch leichter vorhanden $\mathrm{zu}$ sein, wiewohl Winckel seinen Fall als Follikelcyste ansieht. Die massenhaften Wucberungen der Epithelzellen in der fötalen Vagina und in der des Nengeborenen können wohl die Verklebung der Falten vermrsachen; ich selbst hatte eimnal, als ich mich gerade mit dieser Arbeit beschäftigte, im anatomischen Laboratorium des Herrn Professor Schwalbe die Gelegenheit, in einem Sagittalschnitt eines Neugeborenen die Vagina zu untersuchen, deren oberer Theil um die Portio herum sehr stark erweitert war, während die übrige Scheide einen verhältnissmässig engen Canal darstellte. Herr Professor Schwalbe hatte die Giite, mir mitzutheilen, dass der erweiterte Theil vor der Anfertigung des Präparates mit einer flïssig breiigen Nasse angefiillt war. Als ich später diesen Inhalt mikroskopisch untersuchte, fand ich darin nur Zellmassen. Es kann in diesem Falle eine Verengerung des Scheidenrohres wohl durch die Verklebung einiger Schleimhautfalten im mittleren Drittel der Scheide entstanden sein, sodass sich die abgestossenen Epithelzellen hauptsächlich im oberen Drittel angeliäuft und dadurch die Erweiternng dieses Theiles hervorgerufen hatten.

Was die anderen Cysten betrifft, so liegt die Vermuthung nale, dass sie in ganz anderer Weise als die schon beschriebenen entstanden sind. Wie schon erwähnt, konnte man an der Cysteninnenfläche keinen regelmässigen Epithelialüberzng nachweisen, es waren entweder beliebige Zellhaufen oder solche, die eine Zellschicht darstellend auf dem Höhlenrande gelagert waren und in keiner Weise eine epitheliale Anordnung zeigten. Ausser den Cystchen fanden sich im IV. Falle noch zahlreiche verschieden grosse, circlımscripte Zellanlıäufungen, welche jedoch in der Gesammtheit viel kleiner als die vorhandenen Cysten sind. Wir können hier das Wachsthım der Föllikel und schliesslich die Unwandlung derselben in die Cysten deutlich verfolgen; denn wir finden zunächst ganz kleine Heerde von Zellen, dann grössere von gleicher Beschaffenheit, endlich noch grössere, die im Innern schon Höhlenbildung zeigen. Dass diese follikelartigen Gebilde wesentlich von pathologischen Zuständen abhängig sind, beweist die gleichzeitig vorhandene Zelleninfiltration der ïbrigen Mucosa, besonders um die circumscripten Zellheerde. Die Cysten bildeten sich also wohl aus den beschriebenen Zellenanhäufungen in der Mucosa der Scheide. Ob solche Cysten in ilnem späteren Stadium einen regelmässigen Epithelïberzug an der Innenfliche erhalten können, bleibt vor der Hand dahingestellt.

Man darf nach den obigen Betrachtungen wohl nicht eine und dieselbe Ursache fïr die Entstehung aller Scheidencysten hinstellen, es ist verständlich geworden, dass die beiden oben genannten $1 \mathrm{l}^{-}$sächlichen Momente in einer und derselben Vagina zu Cystenbildungen die Veranlassung geben, wie dies aus dem I. Falle ersichtlich ist. Man fand hier nämlich mikroskopische cystöse Ränue, welche aber eine ganz andere Structur als die grosse Cyste zeigten, deren Entstehung wohl einer Zerspaltung von Zellhaufen zngeschrieben werden musste. 